\title{
Proton induces apoptosis of hypoxic tumor cells by the p53-dependent and p38/JNK MAPK signaling pathways
}

\author{
KHEUN BYEOL LEE ${ }^{1}$, KYE-RYUNG KIM ${ }^{2}$, TAE-LIN HUH ${ }^{1}$ and YOU MIE LEE ${ }^{1}$ \\ ${ }^{1}$ School of Life Sciences and Biotechnology, College of Natural Sciences, Kyungpook National University, Daegu 702-701; \\ ${ }^{2}$ Proton Engineering Frontier Project, Korea Atomic Energy Research Institute, Deajeon, Korea
}

Received June 11, 2008; Accepted July 26, 2008

DOI: 10.3892/ijo_00000115

\begin{abstract}
Tumor hypoxia is a main obstacle for radiation therapy. To investigate whether exposure to a proton beam can overcome radioresistance in hypoxic tumor cells, three kinds of cancer cells, Lewis lung carcinoma (LLC) cells, hepatoma HepG2 and Molt-4 leukemia cells, were treated with a proton beam $(35 \mathrm{MeV}, 1,2,5,10 \mathrm{~Gy})$ in the presence or absence of hypoxia. Cell death rates were determined $72 \mathrm{~h}$ after irradiation. Hypoxic cells exposed to the proton beam underwent a typical apoptotic program, showing condensed nuclei, fragmented DNA ladders, and poly-ADP-ribose polymerase (PARP) cleavage. Fluorescence-activated cell sorter analysis revealed a significant increase in Annexin-Vpositive cells. Cells treated with the proton beam and hypoxia displayed increased expression of p53, p21 and Bax, but decreased levels of phospho-Rb, Bcl-2 and XIAP, as well as activated caspase- 9 and -3 . The proton beam with hypoxia induced cell death in wild-type HCT116 cells, but not in a p53 knockout cell line, demonstrating a requirement for p53. As reactive oxygen species (ROS) were also significantly increased, apoptosis could also be abolished by treatment with the anti-oxidant N-acetyl cysteine (NAC). P38 mitogenactivated protein kinase (MAPK) and c-Jun N-terminal kinase (JNK) were activated by the treatment, and their respective DN mutants restored the cell death induced by either proton therapy alone or with hypoxia. In conclusion, proton beam treatment did not differently regulate cancer cell apoptosis either in normoxic or hypoxic conditions via a p53-dependent mechanism and by the activation of $\mathrm{p} 38 / \mathrm{JNK}$ MAPK pathways through ROS.
\end{abstract}

Correspondence to: Dr You Mie Lee, School of Life Sciences and Biotechnology, Department of Natural Sciences, Kyungpook National University, Daegu 702-701, Korea

E-mail: lym@knu.ac.kr

Key words: proton beam, spread out Bragg peak, hypoxia, reactive oxygen species, p53, p38/JNK MAP kinases

\section{Introduction}

Tumor hypoxia is known to be a main obstacle for conventional radiation therapy. Cells in the hypoxic region of the tumor are resistant to radiation and more likely to metastasize aggressively $(1,2)$. To overcome this resistance, many clinical trials are attempting to sensitize hypoxic tumor cells using nitroimidazol derivatives (3), or genetic approaches to inhibit hypoxia inducible factor (HIF)-1 $\alpha$ (4-6) or Ras (7). While p53, caspase-3 and JNK/SAPK are associated with cell cycle arrest and apoptosis that occur as a result of $\gamma$-irradiation in cancer cells, hypoxia-induced apoptotic resistance might be attributed to a general suppression of caspase-3 and JNK/SAPK in the cells (8).

Proton beam therapy has been applied to tumor patients in clinical studies for many years $(9,10)$. When these charged particles enter the patient's body, their specific energy per unit of length deposited along the track (linear energy transfer, LET) increases with decreasing particle velocity, giving rise to a sharp peak of ionization near the end of the range (the Bragg peak). Optimization of the dose needed to induce the cell death of various cancer cells at the Bragg peak is required to reduce the side effects and maximize the benefits of the therapy (11). In the spread-out Bragg peak (SOBP), particles show a broad energy spectrum across the range of sample depths. The relative biological effectiveness (RBE) value for proton beam therapy with SOBP relative to $\gamma$-rays has been evaluated in vitro and in vivo and ranges from 0.7 to 2.1 (12). It is important to pinpoint the actual RBE value, this will permit the determination of equivalent doses of protons and $\gamma$-rays to the tumor region. However, the ability of proton beam therapy to overcome hypoxia-induced resistance to tumor cell death has not yet been assessed, nor the molecular mechanisms that determine the efficacy of this treatment under hypoxic conditions.

Most hadron treatments of charged particles (i.e., proton) or photons (i.e., $\gamma$-ray) are known to induce DNA damage in tissues. Little is known, however, about the differences in death signaling pathways that underlie hadron-induced cell death. Cell death induced by a low dose of proton beams was recently identified as apoptosis rather than necrosis or autophagy (13). In the extrinsic death-inducing signaling pathway, procaspase- 8 undergoes auto-proteolytic activation and allows induction of proapoptotic Bcl-2 family proteins. 
This can lead to the triggering of enzymatic activity of downstream effectors such as caspase-3 and -7 (14). In response to several different death stimuli, such as reactive oxygen species (ROS) or increased intracellular calcium concentration, mitochondrial permeabilization occurs and cytochrome $\mathrm{c}$ is released from the mitochondria into the cytosol where it binds to apaf-1. The apaf-1/cytochrome c complex recruits and activates caspase-9 in the presence of ATP (15). Inhibitors of apoptosis (IAPs) prohibit caspase-3 activation by caspase- 9 (intrinsic pathway). Thus, multiple stimuli with or without the classical Bcl-2 family proteins converge on the mitochondria and trigger the release of proapoptotic molecules to initiate the death cascade (16).

The tumor suppressor protein p53 is a major downstream effector of the DNA-damage pathway for cell cycle arrest and apoptosis. p53-dependent signaling results in G1 arrest and is mainly mediated by transcriptional activation of p21 (17). The activation of p53 is typically induced by DNA damaging stresses, and ROS appear to be generated downstream of p53-induced caspase activation and apoptosis (18). Recently, p38 MAPK was suggested to be a sensor of ROS levels and a negative regulator of malignant transformation (19). It has also been reported that p38 MAPK is induced during p53-dependent cell cycle arrest (20) and that JNK- and p38-mediated phosphorylation of p53 may play a role in their pro-apoptotic action of $\mathrm{p} 53$ response $(21,22)$. It is commonly thought that ROS may be involved in $\gamma$-irradiationinduced apoptosis of cancer cells as well as normal cells $(23,24)$.

The present study investigated whether proton beam therapy can induce cancer cell death under hypoxic conditions in vitro and revealed the mechanism by which tumor cell apoptosis occurs in response to the treatment.

\section{Materials and methods}

Cell culture and hypoxia treatment. Lewis lung carcinoma cells (LLC), Molt-4 human leukemia cells and HepG2 human hepatocellular carcinoma cells were maintained in DMEM supplemented with $10 \%$ fetal bovine serum (heat-inactivated, Hyclone, Logan, UT) and $1 \mathrm{x}$ antibiotics $[100 \mathrm{U} / \mathrm{ml}$ penicillin and $100 \mu \mathrm{g} / \mathrm{ml}$ streptomycin, from Invitrogen (Carlsbad, $\mathrm{CA})]$ at $37^{\circ} \mathrm{C}$ in a humidified atmosphere containing $5 \%$ $\mathrm{CO}_{2}$. For induction of hypoxia, $160 \mu \mathrm{M}$ desferrioxamine was applied $4 \mathrm{~h}$ before proton beam radiation. During beam treatment, we did not change the media, closed the culture bottle cap and irradiated with protons or $\gamma$-rays.

Proton beam irradiation and dosimetry. $35 \mathrm{MeV}$ protons were produced from the MC50 Cyclotron (Scanditronix, Sweden) at the Korea Cancer Center Hospital (Seoul, Korea). Anchored cells were irradiated in a $12.5 \mathrm{~cm}^{2}$ flask or 96-well plate filled with media, and placed on a beam stage. Flasks were oriented such that the growth surface was orthogonal to the horizontal beam entering from the top of the flask. A mono-energetic proton beam cannot be applied for cancer cells because the Bragg peak is too narrow to give a uniform dose to a tumor of any significant depth. Thus, SOBP dose distribution was created by traversing a rotating range modulator designed to obtain a uniform dose distribution to an indicated depth in cells plated and media (25; Fig. 2A). It was assumed that the thickness of the cell monolayer was between 3-6 $\mu \mathrm{m}$ and that of media was $1 \mathrm{~cm}$. Thus dose distribution by SOBP was enough to target live cells. The average dose rate was $2.31 \mathrm{~Gy} / \mathrm{sec}$. Radiochromic film (GAF-MD55) was used as an in situ measuring tool of the dose at each beam irradiation. Irradiation with $\gamma$-rays was performed using ${ }^{137} \mathrm{Cs}$ in IBL 437C $\gamma$-irradiator (CIS Bio International, France) at Kyungpook National University Hospital in Daegu. The dose range for irradiation was from 1 to $100 \mathrm{~Gy}$ with an average dose rate of $1 \mathrm{~Gy} / \mathrm{min}$. All irradiations were performed in air at room temperature.

Cell viability assay. Cell viability was measured with MTT (3-(4,5-dimethylthiazol-2-yl)-2,5-diphenyltetrazolium bromide). For floating leukemia cells, viable cells were measured by Cell Counting Kit-8 (DOJINDO, Japan) at $450 \mathrm{~nm}$ using a microplate reader at three days after proton beam irradiation. Each condition was tested in quadruplicate, the mean \pm SD was calculated, and the fold induction compared to the control cells was determined.

Expression vectors and transient transfection. Transfection was performed using the Lipofectamine 2000 reagent (Invitrogen), according to the manufacturer's instruction. The dominant negative (DN) construct of ERK, JNK-1 and DN p38 MAPK cloned into pCDNA3 or pCMV5 were kindly provided by Dr Aree Moon (College of Pharmacy, Duksung Women's University, Seoul, Korea).

Western blot analysis. Cells were harvested with a lysis buffer (iNtRon Korea), and equal amounts of cellular proteins were subjected to SDS-PAGE. Proteins were transferred to a nitrocellulose membrane and the blots were probed with anti-p53 (Upstate), anti-PARP-1 (Santa Cruz), anti-caspase-3, -7, -9 (Stessgenes), anti-Bcl-2, anti-Bax (Cell Signaling), anti-XIAP (Stressgenes), anti-survivin (Stressgenes), antiphospho-p38, anti-phospho-JNK/SAPK, and anti-phosphoERK1/2 (Cell Signaling) antibodies.

FITC-Annexin V/PI staining and cell cycle analysis. Apoptosismediated cell death and cell cycles of tumor cell were examined using a double staining method with FITC-labeled Annexin-V (Invitrogen) and propidium iodide (PI, SigmaAldrich) as previously described (26). Fluorescent cells were analyzed with a flow cytometer, the Coulter Elite ESP Cell Sorter (Beckman).

DAPI staining and $\gamma H 2 A X$ immunostaining for fragmented nuclear DNA. Cells were harvested, washed, and fixed with $4 \%$ paraformaldehyde for $30 \mathrm{~min}$ at $\mathrm{RT}$ and then resuspended in PBS and stained with DAPI solution $(1 \mu \mathrm{g} / \mathrm{ml}$; Sigma, St. Louis, MO, USA) or immunostained with antihuman H2AX (phospho-S139) (Abcam, Cambridge, MA) and the secondary goat anti-rabbit FITC antibody (Santa Cruz). The slides were examined under fluorescence microscopy with magnification $\mathrm{x} 400$.

Caspase activity analysis. Cells were lysed with a lysis buffer (iNtRon Biotech) and the cell lysate was incubated with 
A

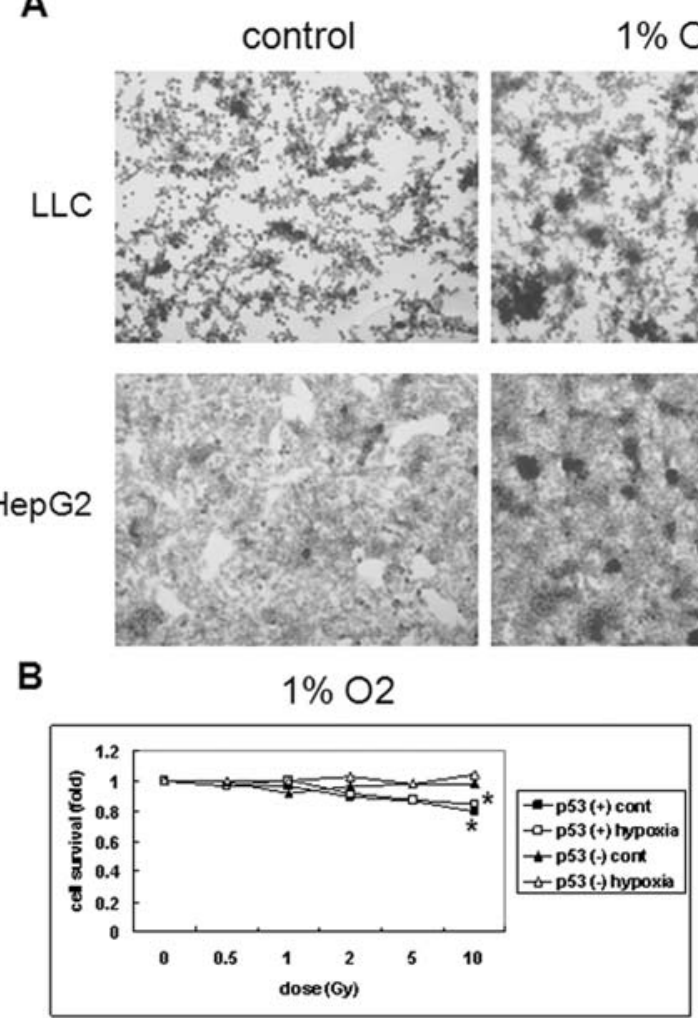

$1 \% \mathrm{O} 2$
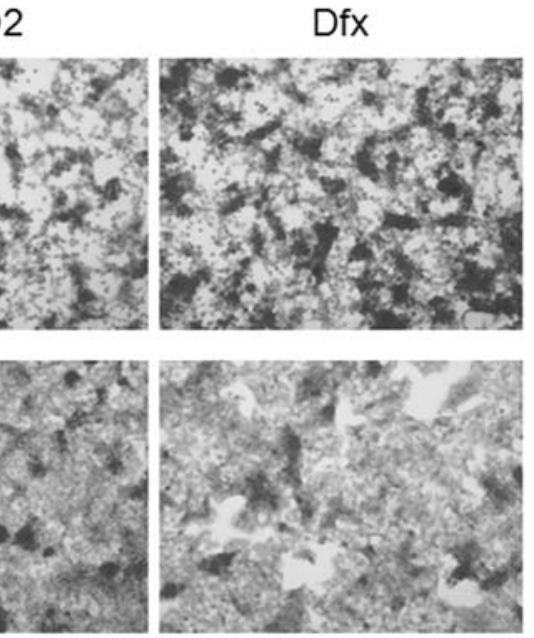

Dfx

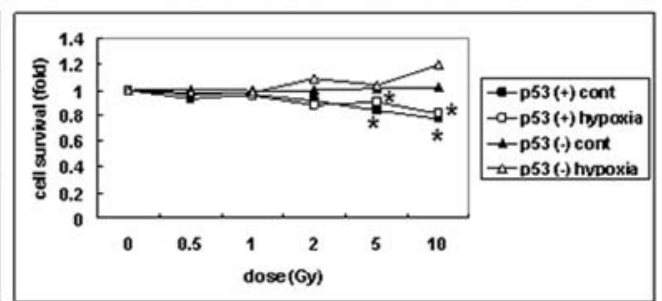

Figure 1. Chemical hypoxia is not different from physical hypoxia to induce hypoxic condition in cells. A, Determination of hypoxia in cells in hypoxic chamber $\left(1 \% \mathrm{O}_{2}\right)$ or dfx treatment. LLC and HepG2 cells were seeded onto 4-well plate in $1 \times 10^{5}$ density and after $24 \mathrm{~h}$ cells were treated with pimonidazol $\mathrm{HCl}(200 \mathrm{mM})$ and incubated with $1 \% \mathrm{O}_{2}$ condition or with dfx $(160 \mathrm{mM})$ for $2 \mathrm{~h}$. After that cells were fixed with $4 \%$ paraformaldehyde, immunohistochemistry with anti-Hypoxyprobe-1 antibody (Chemicon, MA) was performed and detected with ABC kit. Dark color indicates the hypoxic cells in culture plate. B, HCT 116 colon cancer cells (with or without p53) were treated with $1 \% \mathrm{O}_{2}$ condition or with dfx for $2 \mathrm{~h}$ and then irradiated with proton. At $72 \mathrm{~h}$ after irradiation, cell viability was determined with MTT assay.

caspase substrate, Ac-DEVE-pNA (Caspase-3 substrate 1, Calbiochem, San Diego, CA) at $37^{\circ} \mathrm{C}$. Absorbance at $405 \mathrm{~nm}$ was measured every $10 \mathrm{~min}$ by a spectrophotometer. Caspase-3 activity was determined to be the average slope of increasing absorbance plotted as a function of time (within $60 \mathrm{~min}$ ) using a linear regression across duplicate experiments.

ROS measurement. Cells were collected at $4 \mathrm{~h}$ after proton beam irradiation and the intracellular ROS production was measured using an oxidant-sensitive fluorescent probe, DCFH-DA, and fluorescence-activated cell sorter (FACS) analysis, using a FACSCalibur flow cytometer (BectonDickinson). The intracellular hydrogen peroxide concentration was determined as described (27).

Statistical analysis. The significance of differences between data sets obtained for cell survival, capspase 3 activity, and DCF fluorescence for ROS generation was determined by ANOVA. The level of significance was set at $\mathrm{p}<0.01$ or $\mathrm{p}<0.05$ as indicated in the figure legends.

\section{Results}

Proton beam therapy with SOBP induced tumor cell death in cancer cells, even under hypoxic conditions. The ability of proton beams to induce cell death has been investigated in three cancer cell lines: LLC, HepG2, and Molt-4 cells. To induce the hypoxic condition, the cells were treated with desferrioxamine $(\mathrm{dfx}, 160 \mu \mathrm{M}) 4 \mathrm{~h}$ before and during beam exposure. To verify whether dfx could induce hypoxic conditions similar to physical hypoxia, we used Hypoxyprobe-1 (pimonidazole $\mathrm{HCl}$, Chemicon) to detect the pimonidazole adduct which is produced only in hypoxic cells. We found that dfx generated hypoxic stress in cells to the same degree as physical hypoxia $\left(1 \% \mathrm{O}_{2}\right.$ concentration in hypoxic chamber) (Fig. 1A). Physical hypoxia and dfx induced the same effects on cancer cell death in combination with proton therapy (Fig. 1B). We therefore used $\mathrm{dfx}$ as the hypoxic agent because physical hypoxia could be overcome by the cellular reoxygenation that might occur by the moving a culture flask to the radiation room, whereas dfx would remain present in media during irradiation, yielding consistently hypoxic conditions.

When cells were treated with a single dose of the proton beam (1-10 Gy), the cell death rate at $72 \mathrm{~h}$ after irradiation showed a dose-dependent increase in LLC and HepG2 cells both normoxic and hypoxic conditions ( 20-40\%, Fig. 2B). Interestingly, the cell survival rate in the hypoxic group was similar to that of the normoxic group for both LLC and HepG2 cells, suggesting that proton beams might be equally effective under both conditions. However, Molt-4 leukemia cells were not responsive to proton irradiation under normoxia or hypoxia (cell death rate, $\sim 7-11 \%$ ). We then repeated these 
A

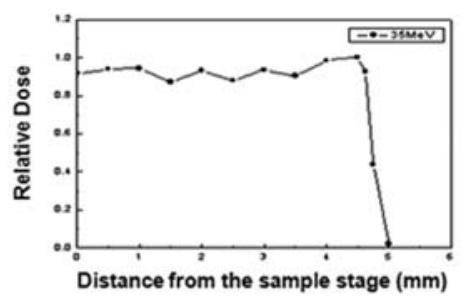

B
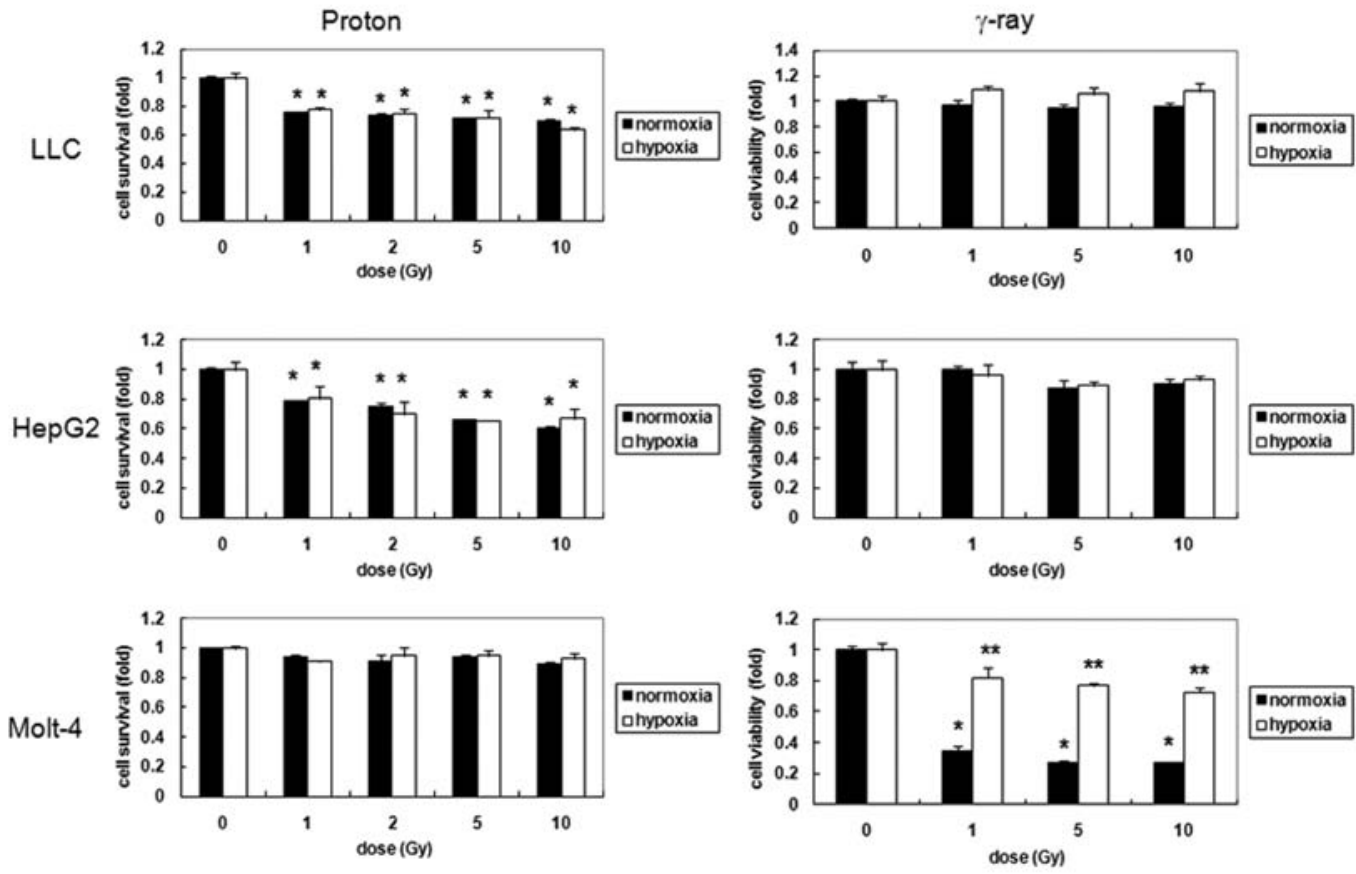

Figure 2. A measured dose with SOBP in a culture plate versus distance from the sample stage, and the effects of proton therapy on three different cancer cell lines. A, The distance 0 was estimated as the entrance point to the plastic plate on which cells were plated. Cell viability was determined by MTT (LLC and HepG2) or CCK-8 assay (Molt-4) under normoxic or hypoxic conditions. Results from three separate experiments are presented as fold induction of control (no irradiation). The solid bar indicates the survival rate at $72 \mathrm{~h}$, mean $\pm \mathrm{SD}(\mathrm{n}=5)$. ${ }^{*} \mathrm{p}<0.01$ compared to control; ${ }^{* *} \mathrm{p}<0.01$ as compared to normoxic cells.

experiments with the same doses of $\gamma$-ray used in place of proton irradiation, in order to determine the sensitivity of these cancer cells to $\gamma$-rays. Surprisingly, $\gamma$-ray treatment induced severe cell death (75\%) only in Molt-4 cells at doses between 1 to 10 Gy and not in LLC and HepG2 cells (0-11\%) (Fig. 2B, right panel). Moreover, hypoxic resistance was observed in Molt-4 cells treated with $\gamma$-rays, i.e., even at $10 \mathrm{~Gy}$ of $\gamma$-rays, hypoxic cells were more likely to survive (76\%) than cells in the normoxic condition (25\%) (Fig. 2B, right panel). These results showed that proton beams induced cell death even under hypoxic conditions in LLC and HepG2, and that sensitivity to proton and $\gamma$-ray treatment was different among cancer cell lines.

Cell death induced by proton beam in hypoxia is apoptosis and increased sub-G1 population. To initially investigate whether the cell death induced by proton beams in HepG2 and LLC cells is processed by an apoptotic mechanism, cell morphology was observed after irradiation. As shown in Fig. 3A, DAPI staining showed that, as compared to the control, proton-treated cells had a condensed or large mass in their nuclear substance or detached spots with a clear margin of membrane. Nuclear DNA was fragmented, condensed and unequally divided (white arrows). To confirm this observation, HepG2 cells were immunostained with an antibody against phospho- $\gamma-\mathrm{H} 2 \mathrm{AX}$, a marker for persistent DNA damage. Proton treatment with or without hypoxia increased number of phospho- $\gamma-\mathrm{H} 2 \mathrm{AX}$ positive foci in the nucleus (black arrows). Thus, these results imply that proton-irradiated cells exhibit the apoptotic cell death pattern under either normoxic or hypoxic regimes. The Annexin V-positive compartment was increased from 2.77 to $77.69 \%$ or $68.84 \%$ by proton treatment with or without hypoxia, respectively (Fig. 3B, right two sections of diagram). In addition, hypoxia decreased an early-stage apoptotic fraction (right lower) but increased a median apoptotic fraction (right upper). The late stage apoptotic and necrotic cell populations were decreased by the proton treatment (Fig. 3B, left upper). These results suggest that proton therapy induced cancer cell death by an apoptotic death mechanism, independent of oxygen status.

The population of sub-G1 LLC cells, described as M1 from the cell cycle profile, typically contains cell debris and apoptotic cells. Compared to the control group, the irradiated cells showed a $38.2 \%$ increase in the sub-G1 phase, whereas hypoxic cells treated with proton beams demonstrated an increased sub-G1 population of $52 \%$. The numbers of cells in 
A

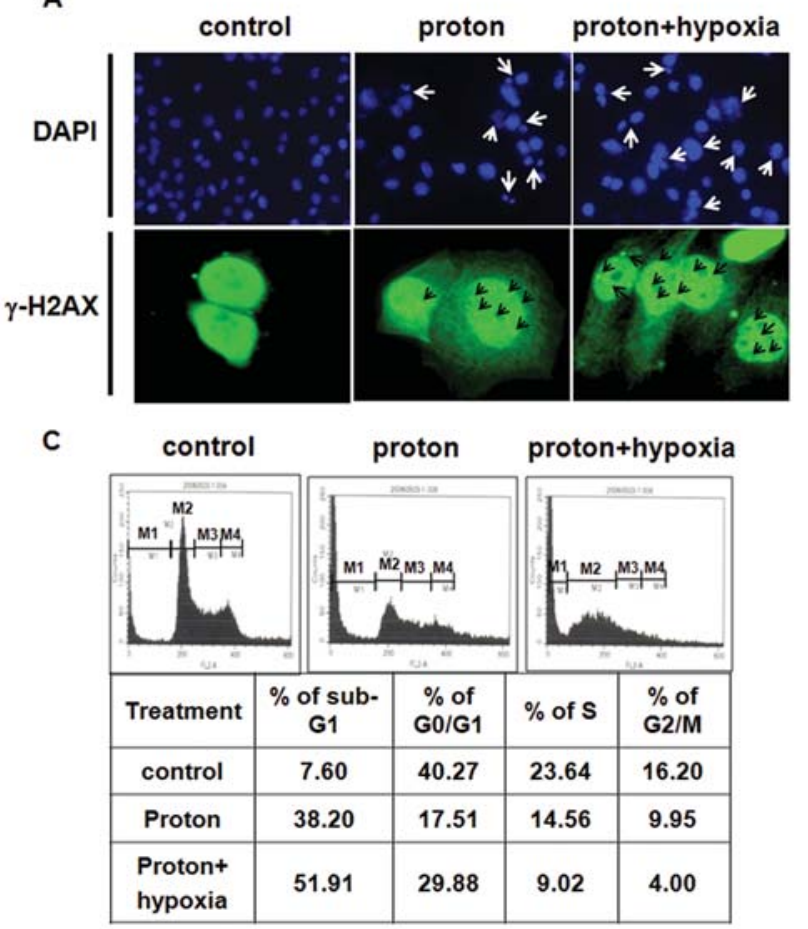

B

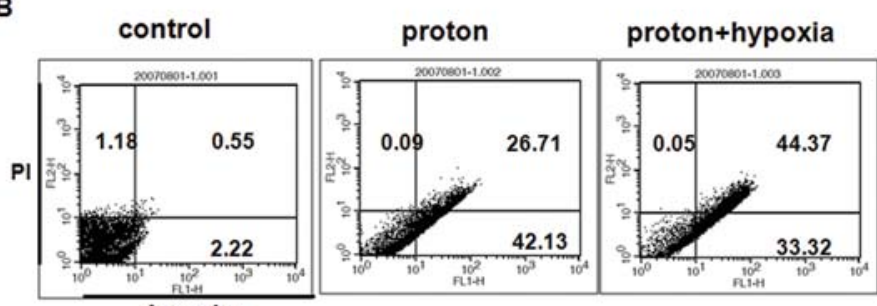

D

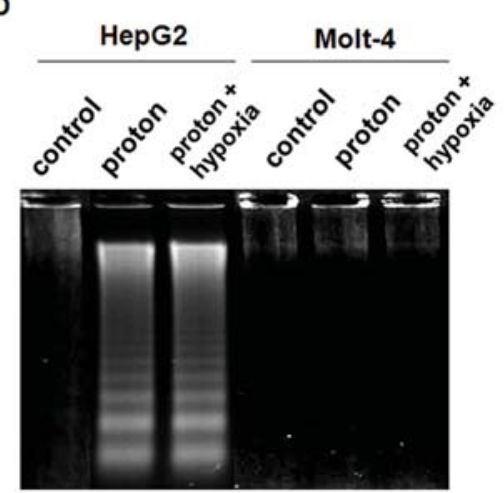

$\mathbf{E}$

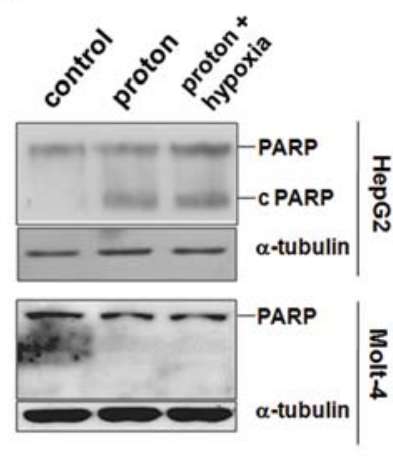

Figure 3. Proton induces apoptotic cell death in proton-sensitive cells. A, HepG2 cells were treated with or without dfx (160 $\mu \mathrm{M})$ and proton irradiated (10 Gy). DAPI staining and phosphorylated $\gamma-\mathrm{H} 2 \mathrm{AX}$ immunostaining was performed $24 \mathrm{~h}$ after proton beam treatment. Arrows indicate fragmented and segregated nuclear DNA (x200). Note the clear fluorescent spots (arrows) in the nucleus indicating double strand breaks in phosphorylated $\gamma$-H2AX immunostained cells (x400). B, Twenty-four hours after irradiation with proton, LLC cells were stained with FITC-Annexin V to detect cell death phenomena. C, After $24 \mathrm{~h}$ of irradiation, cells were stained with PI for cell cycle analysis. The cell cycle phases are indicated as M1 (apoptosis/necrosis, sub-G1), M2 (G0/G1), M3 (S), and M4 (G2/M). D, DNA fragmentation assay was performed at $24 \mathrm{~h}$ after proton irradiation in both HepG2 and Molt-4 cells. E, PARP cleavage was observed by Western blot analysis.

S-phase and G0/G1 phase were decreased and G2/M phase cells were slightly attenuated after proton-irradiation, as in hypoxic cells (Fig. 3C). In HepG2 cells similar cell cycle profiles were observed (data not shown).

When we performed the DNA fragmentation assay, we observed that proton therapy induced clear DNA fragmentation in both normoxic and hypoxic HepG2 cells, but did not induce any fragmentation in Molt-4 cells (Fig. 3D). Moreover, cleaved PARP molecule, a marker for apoptotic cell death, was observed in response to proton treatment in HepG2 cells but not in Molt-4 cells under normoxia or hypoxia (Fig. 3E). These results indicate that in proton-sensitive LLC and HepG2 cells, cell death might occur via an apoptotic mechanism and that proton therapy may bypass hypoxic resistance.

Proton therapy induced apoptosis under hypoxic conditions by a p53-dependent mechanism that included activation of caspases. To investigate the mechanisms by which proton treatment induces apoptotic cancer cell death, we performed Western blot analysis with antibodies against apoptosis signaling proteins. As shown in Fig. 4A, proton therapy caused slight increases in p53 and induction of downstream targets of p53, such as p21 and Bax. Co-treatment with hypoxia increased the level of p53 as well as p21 and Bax. In contrast, cell survival factor, Bcl-2, phospho-Rb, and XIAP were downregulated by proton therapy with or without hypoxia. We next examined the expression of caspase family proteins in HepG2 cells. The level of cleaved caspase-3 was increased by proton treatment with or without hypoxia. Pro- caspase-9 was dramatically decreased in proton-treated cells under hypoxic regimes as well as by proton therapy alone, but pro-caspase- 8 was not changed (Fig. 4A). In contrast, in Molt-4 cells irradiated with protons with or without hypoxia, there were no changes in p53, Bcl-2, Bax, and caspases levels (Fig. 4B). These results suggest that proton beams induce apoptosis via a p53-dependent pathway, and at least in part through caspase-9/-3 activation in proton-sensitive hypoxic cells.

To confirm the involvement of p53 in proton-induced apoptosis, we used cell lines with different p53 status that were derived from the same genetic background. When HCT116 colon cancer cells with a homozygous null mutation in $\mathrm{p} 53$ (HCT116 $\mathrm{p}^{-/ /}$) were treated with the proton beam, cell death was not induced under normoxic or hypoxic conditions, whereas in HCT116 p53 wild-type (wt) cells, cell death was induced (Fig. 4C). This result indicates that the presence of p53 is crucial for the induction of apoptosis by proton therapy under hypoxic conditions.

Proton beams induced apoptosis through an increase of ROS level under hypoxic conditions. To determine whether the apoptosis induced by proton treatment is mediated by an increase in ROS, we determined the ROS levels at $6 \mathrm{~h}$ postirradiation in LLC and HepG2 cells. Proton irradiation increased ROS generation by $\sim 1.8$-fold compared to controls (Fig. 5A), and the increase was slightly more prominent in hypoxia ( 2.1-fold). When we pretreated with $\mathrm{N}$-acetyl cysteine (NAC) as a scavenger of ROS for $24 \mathrm{~h}$, the decreased 

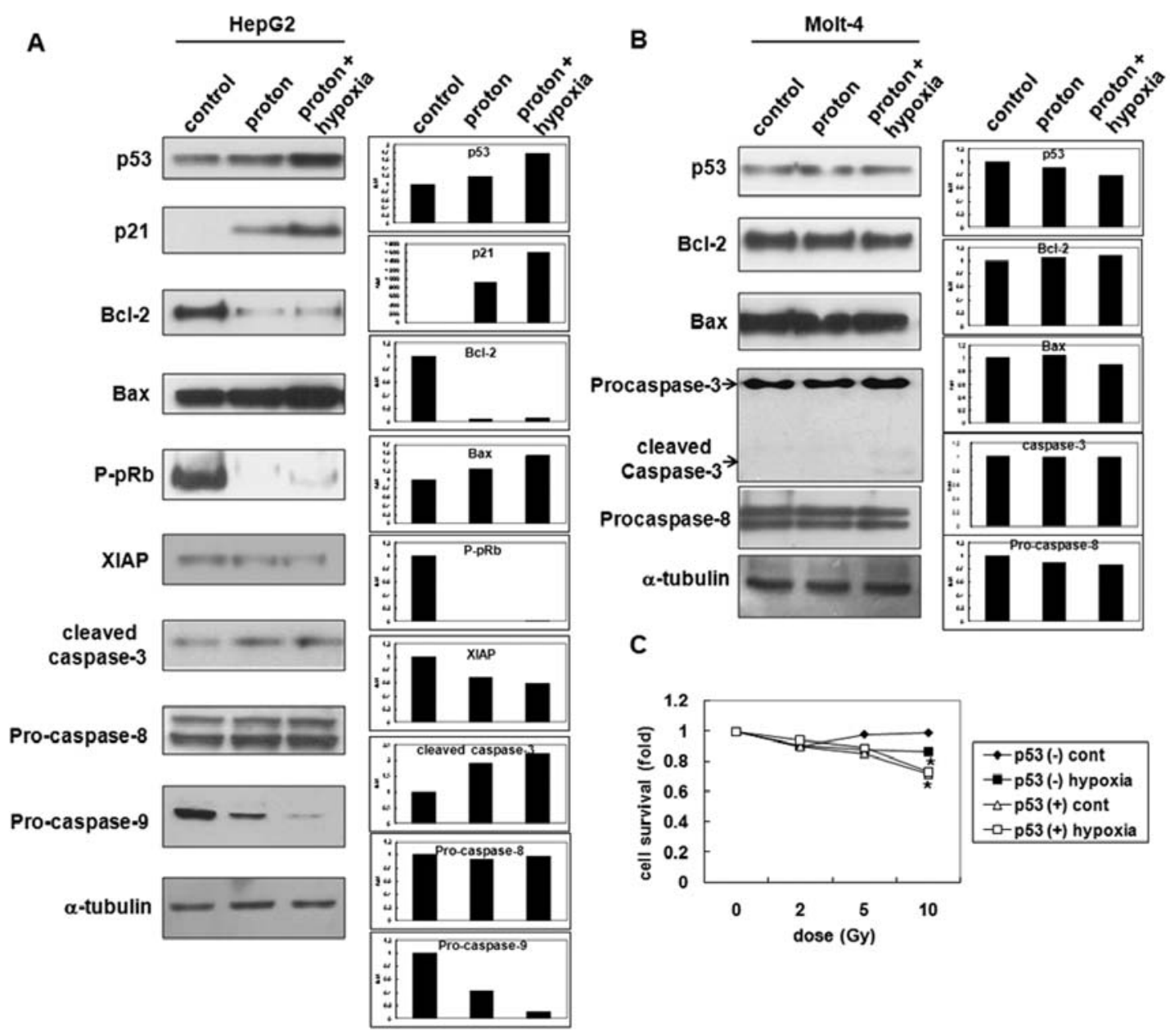

Figure 4. Western blot analysis in proton-irradiated cancer cells. A, HepG2 cells irradiated with proton (10 Gy) were incubated for $24 \mathrm{~h}$, collected and lysed for Western blotting. The graphs on the right are quantifications for each Western band. B, Molt-4 cells were irradiated with proton (10 Gy) and subjected to Western blot analysis. Right solid bar indicates the quantities of each Western band. C, After irradiation with proton (2-10 Gy), cell viabilities at $24 \mathrm{~h}$ were determined for HCT116 p53 wt and p53\% cells. Results are presented as fold induction of control (no irradiation) (n=5). " $\mathrm{p}<0.01$ compared to p53\% cells.

cell survival rate after by proton irradiation recovered from 44-53 to 65-69\% in LLC cells (Fig. 5B). To further confirm the involvement of ROS in proton-induced apoptosis, we analyzed caspase-3 activity. As shown in Fig. 5C, caspase-3 activity was increased over 5 -fold by proton therapy in both normoxia and hypoxia as compared to the control. However, pretreatment with NAC $(5 \mathrm{mM})$ significantly decreased the caspase- 3 activity. The data confirmed that ROS mediate the proton-induced apoptosis via the activation of caspase-3.

To next determine whether p53 activation is upstream or downstream of the ROS generated by proton treatment, we measured ROS levels in the p53 null mutant cell line. Unexpectedly, ROS was increased by proton irradiation in both $\mathrm{p} 53 \mathrm{wt}$ and $\mathrm{p} 53^{-/}$HCT116 cells under normoxia or hypoxia, indicating that $\mathrm{p} 53$ is not essential for generation of ROS (Fig. 5D). This result postulates two possibilities: first, that ROS is the upstream regulator for activation of the p53-dependent pathway by proton treatment, or second, that ROS might partly mediate a p53-independent apoptotic pathway.

Proton beam-activated p38 MAPK and JNK MAPK. To investigate the cell signaling molecules that might be involved in proton-induced apoptosis, we used Western blot analysis to determine the levels of several MAPKs. Proton therapy increased the phosphorylation of p38 MAPK and JNK while it inhibited phospho-ERK (Fig. 6A). Moreover, a kinase involved in cell survival, phospho-Akt was significantly decreased by the proton beam. Oxygen status did not affect proton-induced MAPK activation or survival kinase inhibition (Fig. 6A). In order to verify the involvement of these two stress-induced MAPKs in proton beam-induced apoptosis and ROS generation, we transfected cells with dominant negative (DN) mutants of p38 and JNK MAPKs and examined cell survival rate and ROS generation. DN-p38 and -JNK mutant transfected cells became resistant to the proton beam treatment (Fig. 6B). Control cell survival rate after proton irradiation was $67 \%$, while those of the DN-JNK and -p38 mutants were 84 and $99 \%$, respectively under hypoxia. The effect of DN-p38 MAPK was stronger than that of DN-JNK at all doses of proton radiation, under normoxia or hypoxia.

\section{Discussion}

In the current study, we investigated proton beam-induced tumor cell apoptosis and overcame the typical radioresistance 
A

B
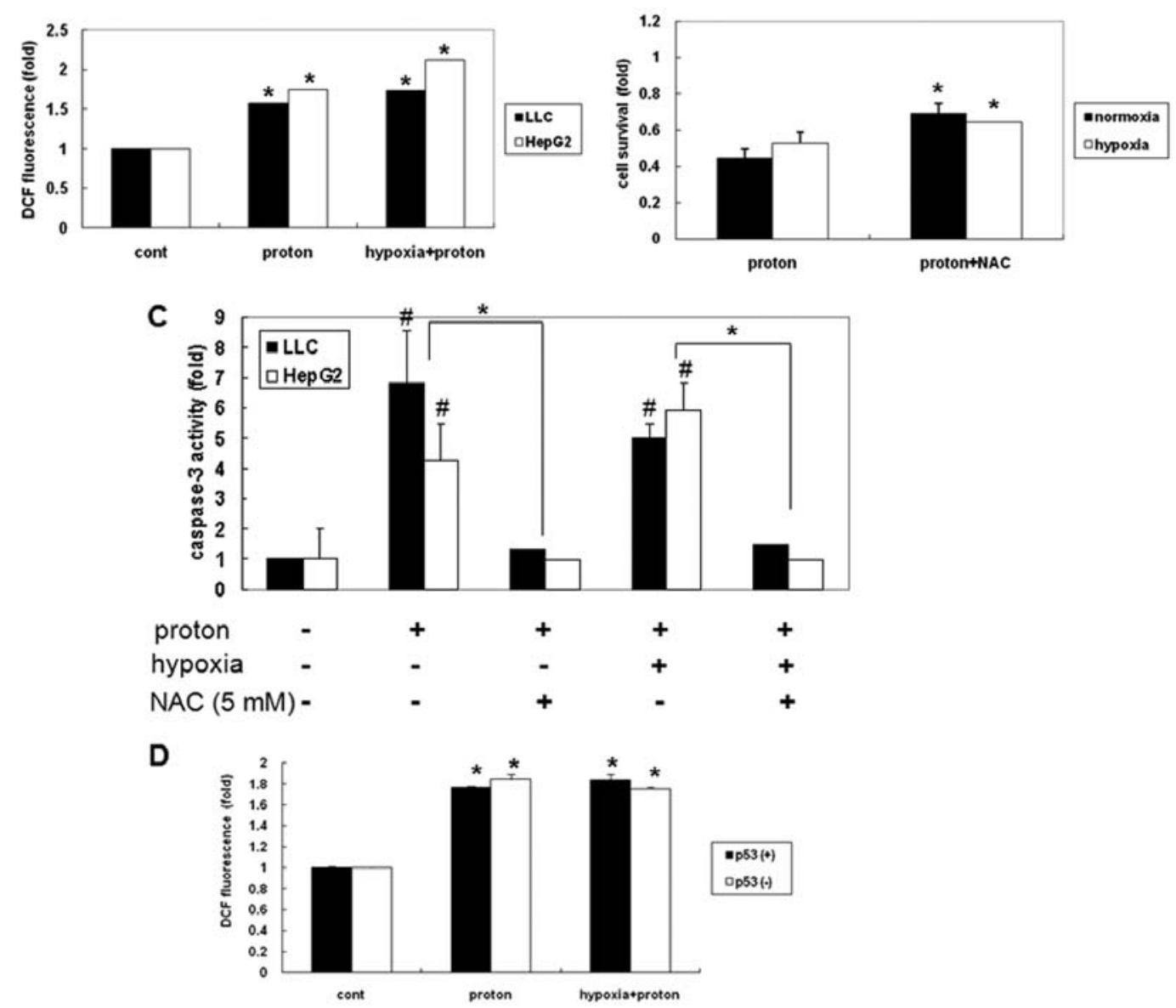

Figure 5. ROS-mediated apoptosis in proton beam-irradiated cancer cells under hypoxia. A, Level of ROS in LLC and HepG2 cells measured by DCFH-DA. $\mathrm{B}$, NAC (N-acetylcysteine. $5 \mathrm{mM}$ ) pretreatment significantly recovered cell survival in the LLC cells irradiated by proton beams at 50 Gy. C, Caspase-3 activity was determined under normoxic or hypoxic conditions in the presence or absence of NAC pretreatment (5 mM). D, HCT116 cells with or without p53 [p53(+), p53(-)] were plated and on the next day irradiated with proton beams (10 Gy) in the presence or absence of dfx (160 $\mu \mathrm{M})$. After $6 \mathrm{~h}$ of irradiation, the quantity of ROS was measured with DCFH-DA. Significances were within $\mathrm{p}<0.01$ (\#) range as compared to control or $(*)$ between two groups.

seen under hypoxic conditions. Typical tumor types treated by proton therapy are melanoma, lung cancer, ocular tumor, breast cancer and nearly solid tumors $(10,28)$. Our experiment used in vitro cell culture systems and sought to investigate the cell viability with a proton beam. Different sensitivities to proton therapy probably derived from the different p53 status of these cell lines, as p53 is mutated in Molt-4 cells and not in LLC and HepG2 cells. This result was consistent with data obtained from HCT116 p53/- cells (Fig. 4C). However, the absolute dependency of p53 status is further to be precisely defined. The question then arises of what causes the different sensitivity to $\gamma$-ray, i.e., why did LLC and HepG2 cells not respond to the same dose of $\gamma$-rays? $\gamma$-ray-induced Molt-4 cell death is caused by p53 accumulation and phosphorylation at serine-392 (29). It has also been determined that sensitivity to $\gamma$-rays is rather irrespective of p53 status (30) and thus concluded that diverse mechanisms might be involved in $\gamma$-rayinduced cell death (31). In our observations, proton therapy clearly induced p53-dependent apoptosis. This may initially result in $\mathrm{G} 1$ phase arrest, as the induction of $\mathrm{p} 21$ by $\mathrm{p} 53$ mediates G1/S checkpoint arrest by inhibition of CDK activity. Proton treatment of hypoxic cells led to increased levels of p21 and activated pRb, suggesting that G1 arrest is a main determinant of apoptosis induction (Fig. 4).

Like other hadron therapy, proton treatment is known to induce DNA damage in cells, but no substantial data has been collected to unveil the specific effects of the therapy or the signaling molecules required for induction of tumor cell death. Two mechanisms have been reported regarding the action of radiation therapy. The first is the canonical apoptotic mechanism that results in cell death within a few hours of radiation. The second is the radiation-induced failure of mitotic progression, which inhibits cancer cell proliferation (19). Our results shown in Figs. 3 and 4 imply that proton therapy could induce both types of cell death pathways. In fact, we did not observe cell death in cancer cells used in this study within a short time period, such as 6 or $12 \mathrm{~h}$ after proton irradiation. We instead saw that an increase in cell death occurred at $24 \mathrm{~h}$ or two or three days after irradiation. However, the early phases of apoptotic cells were observed from FACS data as 42.13 and $33.32 \%$ in proton therapy alone and proton plus hypoxia, respectively $24 \mathrm{~h}$ after irradiation (Fig. 3B). ROS generation and activation of MAPKs were detected within a short time after irradiation (4 and $1 \mathrm{~h}$, 
A
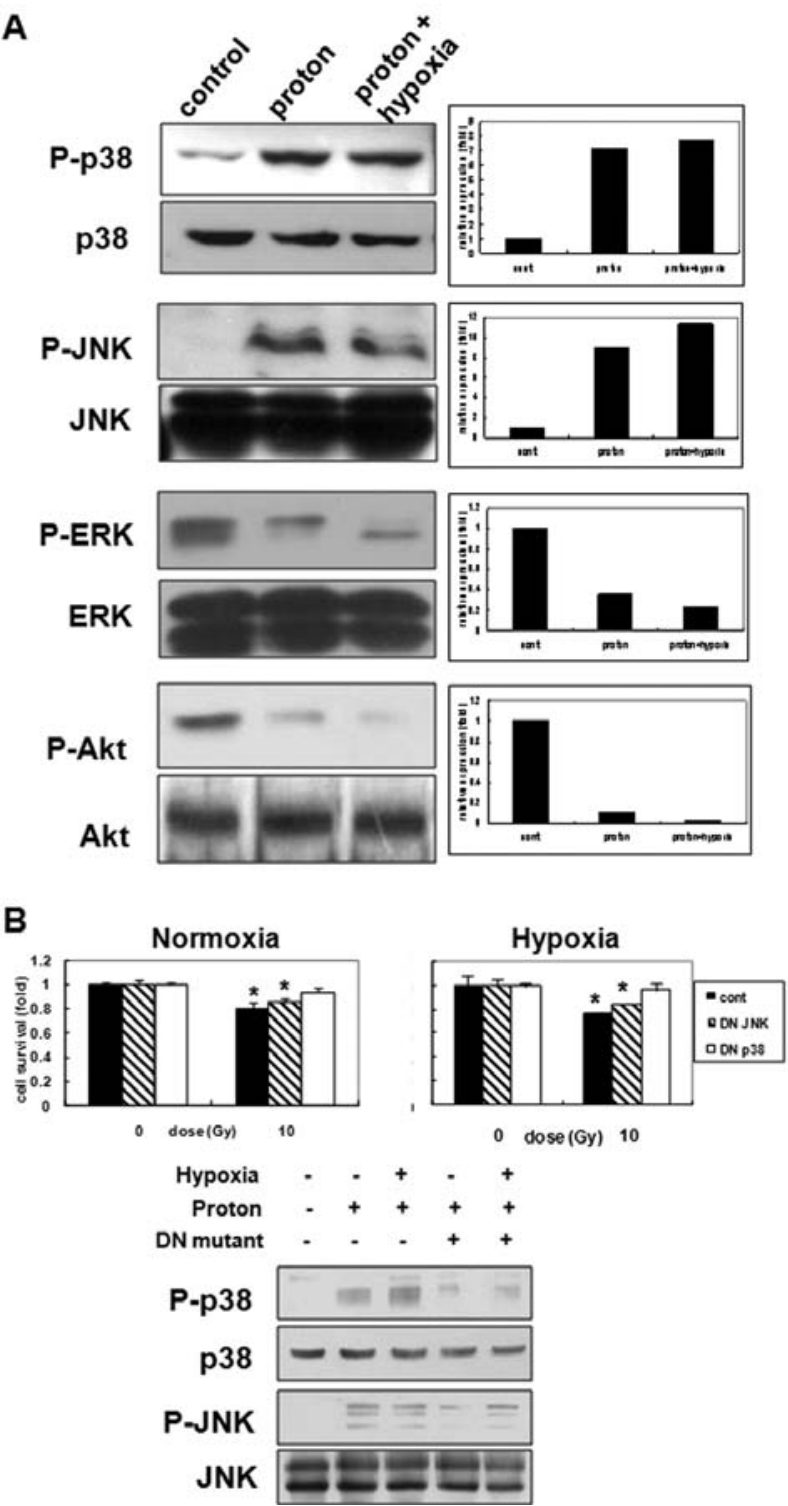

Figure 6. Activation of p38 MAPK- and JNK in proton beam-irradiated LLC and HepG2 cells. A, Proton beams alone or plus hypoxia induced phosphorylation of p38 MAPK, JNK and decreased the amount of detectable p-ERK and p-Akt. The right panel shows the quantitative analysis for each Western blot band, performed with ImageJ. B, HepG2 cells were transfected with DN-p38 MAPK or DN-JNK and irradiated with proton beams (10 Gy) on the following day, in the presence or absence of dfx $(160 \mu \mathrm{M})$. After $24 \mathrm{~h}$, cell viability was determined with MTT assay. The lower panel shows the transfection efficiency of DN mutants, i.e., no activation of p38 or JNK MAPK compared to the total MAPKs. Significance was within $\mathrm{p}<0.01(*)$ range as compared to control.

respectively). We could therefore conclude that the early increase in ROS or p38 and JNK MAPK activation might stimulate the apoptotic signaling cascades to induce the late cell death program as well as early mitotic damage. FACS analysis after Annexin-V-FITC and PI staining and DNA fragmentation gel electrophoresis showed that the cell death patterns are clearly apoptotic, because autophagy cause the nucleosomal ladder formation or typical TUNEL staining (32).

Our results indicate for the first time that proton treatment induces LLC and HepG2 cell death regardless of their oxic status. This is a surprising result because it has been reported that hypoxia diminished the amount of ROS induced by photon treatment (33). However, we show that the increases in ROS due to hypoxia plus proton were more substantial than those due to the proton therapy only (Fig. 5A). A number of groups have reported that ROS levels are increased in hypoxia (34-37) through the insufficiency of antioxidant capacity (38) or mitochondrial complex III (39). Thus, if the mitochondrial ROS induced by hypoxia are added to the proton-induced ROS, the amount of ROS might be increased. However, the ROS generation and total levels might be dependent on the degree and duration of hypoxia and thus to some degree on the experimental model systems (cell types and in vitro or in vivo). Another question is raised as to why the ROS level differs in HepG2 and LLC cells. One possibility is that HepG2 and LLC cells might have different pools of 'redox buffering' systems, including enzymes for catabolism of ROS. Cell lines are known to display differences in activity of ROS scavenger systems, depending on their function, microenvironment, and metabolic status, including the levels of cellular reducing agents, such as NADPH and glutathione (39).

Radiation is usually classified as direct or indirect ionization. When neutrons or protons are used, direct ionization predominates as the atoms of the target, such as DNA, are directly ionized or excited and events that lead to biological changes are initiated. However, Wan et al (40), report that the ROS level induced by X-rays changed as the media volume increased. The high volume of media (200 $\mu \mathrm{l} /$ well in 96-well plates) used in our experiment might contribute to the increases in ROS as observed with X-rays. Under normoxic conditions, the media water would be a main source of ROS, because low-LET radiation less than $2 \mathrm{keV} / \mu \mathrm{m}$ ) such as protons produces sparse ionizations within the irradiated cells and their surrounding environment. Under hypoxic conditions, the mitochondrial ROS (34) may add to or act differently than the ROS generated from media water. Thus, proton therapy might generate ROS by either intracellular or surrounding water ionization, or both, depending on the normoxic or hypoxic condition.

The ROS generation mechanism provides one explanation for why proton beams did not discriminate normoxic or hypoxic cell status. Following generation, ROS signaling might lead to caspase-3 activation. p53 was clearly induced by proton beams in HepG2 and LLC cells, even in hypoxia. While $\gamma$-ray treatment of Molt- 4 cells also induces p53 signaling (29; data not shown) the proton beams did not induce p53 or downstream target proteins in Molt-4 cells (Fig. 4B). The protective effects of the anti-oxidant (NAC) that we observed provide evidence that ROS contribute to the apoptosis of cancer cells (HepG2 and LLC) by proton therapy. Other studies that treated various antioxidants prior to or after proton irradiation indicate that ROS generation is a main cause of adverse effects to animals and of neuronal damage (41). Our Western blot data and deter-mination of the fluorescence content of DCF-DA further indicate that the apoptosis effectors and oxidative stress that mediate proton beam-induced apoptosis are, at least in part, dependent on the activation of p53 and its downstream targets.

From our results, we find that proton beams stimulate p53 and caspase-9, and subsequently caspase-3 (42), and also 
lead to increased oxidative stress, all of which contribute to cell death. Increased ROS may activate p53 in a feed-back loop, but the ROS increase caused by proton therapy is unlikely to be dependent on the p53 genetic background (Fig. 5D). Our results suggest that ROS may act upstream of p53, but in its absence, ROS can slightly induce apoptosis as seen by the death rate of Molt-4 cells (Fig. 2). Moreover, the p53 response might be boosted by JNK and p38 MAPKmediated phosphorylation of p53 $(23,24)$. P38 MAPK is a ROS sensor and acts to inhibit their accumulation, which in turn inhibits tumorigenesis (19). Thus, it is speculated that ROS might be an important upstream regulator of p53 activation, via the JNK pathway and activated p38 MAPK, which are cooperative but distinct pathways to trigger cell death program in cancer cells (Fig. 6).

\section{Acknowledgements}

This work was performed under the User Program of PEFP (Proton Engineering Frontier Project), as a part of the 21C Frontier R\&D Program supported by the Ministry of Science and Technology. Wild-type and p53-null mutant HCT116 cancer cells were provided by Dr Bert Vogelstein (Department of Developmental Neurobiology, St. Jude Children's Research Hospital, Memphis, TN, USA). We thank Dr Jeonghee Yang for critical review and English correction of the manuscript.

\section{References}

1. Horsman MR: Nicotinamide and other benzamide analogs as agents for overcoming hypoxic cell radiation resistance in tumours. Acta Oncol 34: 571-587, 1995.

2. Semenza GL: Intratumoral hypoxia, radiation resistance, and HIF-1 1 . Cancer Cell 5: 405-406, 2004.

3. el Gamoussi R, Stratford IJ and Guichard M: Relationship between intracellular concentration and radiosensitizing effect of pimonidazole and etanidazole on two human melanoma cell lines. Int J Radiat Biol 63: 27-36, 1993.

4. Williams KJ: Enhanced response to radiotherapy in tumours deficient in the function of hypoxia-inducible factor-1. Radiother Oncol 75: 89-98, 2005

5. Chadderton N, Cowen RL, Sheppard FC, et al: Dual responsive promoters to target therapeutic gene expression to radiationresistant hypoxic tumor cells. Int J Radiat Oncol Biol Phys 62: 213-222, 2005.

6. Brunner TB, Gupta AK, Shi Y, et al: Farnesyltrasferase inhibitors as radiation senstitizers. Int J Radiat Biol 79: 569-576, 2003.

7. Brunner TB, Hahn SM, et al: Radiation sensitization by inhibition of activated Ras. Strahlenther Onkol 180: 731-740, 2004.

8. Samuni AM, Kasid U, Chuang EY, et al: Effects of hypoxia on radiation-responsive stress-activated protein kinase, p53, and caspase-3 signals in TK6 human lymphoblastoid cells. Cancer Res 65: 579-586, 2005 .

9. Hashimoto T, Tokuuye K, Fukumitsu N, et al: Repeated proton beam therapy for hepatocellular carcinoma. Int J Radiat Oncol Biol Phys 65: 196-202, 2006.

10. Levin WP, Kooy H, Loeffler JS and De Laney TF: Proton beam therapy. Br J Cancer 93: 849-854, 2005.

11. Bettega D, Calzolari P, Chauvel P, et al: Radiobiological studies on the $65 \mathrm{MeV}$ therapeutic proton beam at Nice using human tumour cells. Int J Radiat Biol 76: 1297-1303, 2000.

12. Paganetti H, Niemierko A, Ancukiewicz M, et al: Relative biological effectiveness (RBE) values for proton beam therapy. Int J Radiat Oncol Biol Phys 53: 407-421, 2002.

13. Di Pietro C, Piro S, Tabbi G, et al: Cellular and molecular effects of protons: apoptosis induction and potential implications for cancer therapy. Apoptosis 11: 57-66, 2006

14. Tsujimoto Y: Cell death regulation by the Bcl-2 protein family in the mitochondria. J Cell Physiol 195: 158-167, 2003.
15. Igney FH and Krammer PH: Death and anti-death: tumour resistance to apoptosis. Nat Rev Cancer 2: 277-288, 2002.

16. Cregan SP, Dawson VL and Slack RS: Role of AIF in caspasedependent and caspase-independent cell death. Oncogene 23: 2785-2796, 2004.

17. Vogelstein B, Lane D and Levine AJ: Surfing the p53 network. Nature 408: 307-310, 2000.

18. Mishra KP: Cell membrane oxidative damage induced by gamma-radiation and apoptotic sensitivity. J Environ Pathol Toxicol Oncol 23: 61-66, 2004.

19. Dolado I, Swat A, Ajenjo N, De Vita G, et al: p38alpha MAP kinase as a sensor of reactive oxygen species in tumorigenesis Cancer Cell 11: 191-205, 2007.

20. Bulavin DV, Demidov ON, Saito S, Kauraniemi C et al: Amplification of PPM1D in human tumors abrogates p53 tumor-suppressor activity. Nat Genet 31: 210-215, 2002.

21. Fuchs SY, Adler V, Pincus MR and Ronai Z: MEKK1/JNK signaling stabilizes and activates p53. Proc Natl Acad Sci USA 95: 10541-10546, 1998.

22. Sanchez-Prieto R, Rojas JM, Taya Y and Gutkind JS: A role for the p38 mitogen-acitvated protein kinase pathway in the transcriptional activation of p53 on genotoxic stress by chemotherapeutic agents. Cancer Res 60: 2464-2472, 2000.

23. Engel RH and Evens AM: Oxidative stress and apoptosis: a new treatment paradigm in cancer. Front Biosci 11: 300-312, 2006.

24. Ozben T: Oxidative stress and apoptosis: impact on cancer therapy. J Pharm Sci 96: 2181-2196, 2007.

25. Lim YK, Park BS, Lee SK, Kim KR and Yang TK: A proton beam irradiation method for a uniform dose distribution over a sample volume. J Kor Phys Soc 48: 777-780, 2006.

26. Van Engeland M, Ramaekers FC, Schutte B and Reutelingsperger CP: A novel assay to measure loss of plasma membrane asymmetry during apoptosis of adherent cells in culture. Cytometry 24: 131-139, 1996.

27. Jiang ZY, Hunt JV and Wolff SP: Ferrous ion oxidation in the presence of xylenol orange for detection of lipid hydroperoxide in low density lipoprotein. Anal Biochem 202: 384-389, 2002.

28. MacDonald SM, De Laney TF and Loeffler JS: Proton beam radiation therapy. Cancer Invest 24: 199-208, 2006.

29. Szkanderova S, Vávorá J, Rézacová M, et al: Gamma irradiation results in phosphorylation of p53 at serine-392 in human T-lymphocyte leukaemia cell line MOLT-4. Folia Biol (Praha) 49: 191-196, 2003.

30. Christophorou MA, Martin-Zanca D, Soucek L, et al: Temporal dissection of $\mathrm{p} 53$ function in vitro and in vivo. Nat Genet 37: 718-726, 2005

31. Soderlund K, Perez-Tenorio G and Stal O: Activation of the phosphatidylinositol 3-kinase/Akt pathway prevents radiationinduced apoptosis in breast cancer cells. Int J Oncol 26: 25-32, 2005.

32. Paglin S, Hollister T, Delohery T, et al: A novel response of cancer cells to radiation involves autophagy and formation of acidic vesicles. Cancer Res 61: 439-444, 2001.

33. Cuisnier O, Serduc R, Lavieille JP, et al: Chronic hypoxia protects against gamma-irradiation-induced apoptosis by inducing bcl-2 up-regulation and inhibiting mitochondrial translocation and conformational change of bax protein. Int J Oncol 23: 1033-1041, 2003.

34. Chandel NS, Maltepe E, Goldwasser E, Mathieu CE, Simon MC and Schumacker PT: Mitochondrial reactive oxygen species trigger hypoxia-induced transcription. Proc Natl Acad Sci USA 95: 11715-11720, 1998.

35. Guzy RD and Schumacker PT: Oxygen sensing by mitochondria at complex III: the paradox of increased reactive oxygen species during hypoxia. Exp Physiol 91: 807-819, 2006.

36. Simon MC: Mitochondrial reactive oxygen species are required for hypoxic HIF alpha stabilization. Adv Exp Med Biol 588: 165-170, 2006.

37. Bell EL, Klimova TA, Eisenbart J, et al: The Qo site of the mitochondrial complex III is required for the transduction of hypoxic signaling via reactive oxygen species production. J Cell Biol 177: 1029-1036, 2007

38. Leonard MO, Kieran NE, Howell K, et al: Reoxygenationspecific activation of the antioxidant transcription factor Nrf2 mediates cytoprotective gene expression in ischemia-reperfusion injury. FASEB J 20: 2624-2626, 2006

39. Cook JA, Gius D, Wink DA, Krishna MC, Russo A and Mitchell JB: Oxidative stress, redox, and the tumor microenvironment. Semin Radiat Oncol 14: 259-266, 2004. 
40. Wan XS, Bloch P, Ware JH, Zhou Z, Donahue JJ, Guan J, Stewart J and Kennedy AR: Detection of oxidative stress induced by low- and high-linear energy transfer radiation in cultured human epithelial cells. Radiat Res 163: 364-368, 2005.

41. Limoli CL, Giedzinski E, Baure J, Rola R and Fike JR: Redox changes induced in hippocampal precursor cells by heavy ion irradiation. Radiat Environ Biophys 46: 167-172, 2007.

42. Wu GS and Ding Z: Caspase 9 is required for p53-dependent apoptosis and chemosensitivity in a human ovarian cancer cell line. Oncogene 21: 1-8, 2002. 